\title{
Theoretical Calculations of Solvent Effects on the Adsorption of Linear Molecules Using the Multilayer Lattice Model
}

\author{
JOHN J. BLADON, WILLIAM I. HIGUCHI, AND ABDULLA M. MOLOKHIA \\ Department of Pharmaceutical Chemistry, University of Michigan, Ann Arbor, Michigan 48104 \\ Received May 31, 1974; accepted March 31, 1975
}

\begin{abstract}
Ash, Everett, and Findenegg's model for multilayer polymer adsorption was modified to handle solvent effects upon adsorption behavior. Some of the assumptions of the model are: (1) the segments of the polymer and the solvent monomer are approximately the same size, (2) the segments of the polymer and the solvent monomer occupy only the lattice points of a given geometrical array (close-packed hexagonal); (3) the energies of interaction between nonbonded segments are angularly independent, are additive in nature, and extend no further than the nearest neighbors; and (4) the surface has a homogeneous interaction energy for each segment type.

The specific polymers examined were the asymmetric dimer $(\mathrm{A}-\mathrm{B})$ and the asymmetric tetramer $(A-B-B-B)$ in a solvent $(C)$.

Parameters required for computer calculations were the nearest neighbor energies of interaction between all combinations of nonbonded segment pairs $(A, A ; B, B ; C, C ; A, B ; A, C$; and $B, C$ ) and interaction energies between the surface and each segment type in each layer near the surface. Analyses were made for the effects of each of these parameters on the surface excess, and on the energy, entropy, and number of each molecular configuration in each layer near the surface.
\end{abstract}

\section{INTRODUCTION}

A model for multilayer polymer adsorption developed by Ash (1), and Ash, Everett and Findenegg (2) predicts total polymer adsorption and the amount of each polymer configuration in each layer near the surface. A description of their model is as follows. The polymer is constructed of two types of segments, arbitrarily labeled $A$ and $B$ (Fig. 1) and the solvent or monomer consists of single segments of type $B$. The segments are of approximately equal size so that they may occupy lattice points arranged in a specific geometrical pattern; close-packed hexagonal arrays were used because this is the most efficient packing of equal-sized spheres. This array results in six contacts for each segment with neighboring segments in the same layer and three contacts with neighboring segments in each of the two adjacent layers. The energies of interaction between nonbonded segments were assumed to extend no further than their nearest neighbors and to be additive in nature. The homogeneous surface had energies of interaction for each segment type and was generally different for the same segment located in different layers.

However, since the entire system could be constructed of only two types of segments, important solvent effects could not be observed. With appropriate modifications, the model can now describe systems in which the polymer is constructed of two types of segments while the solvent consists of a third type.

It is therefore the purpose of this paper to present the results of a systematic analysis of solvent effects upon the adsorption behavior 


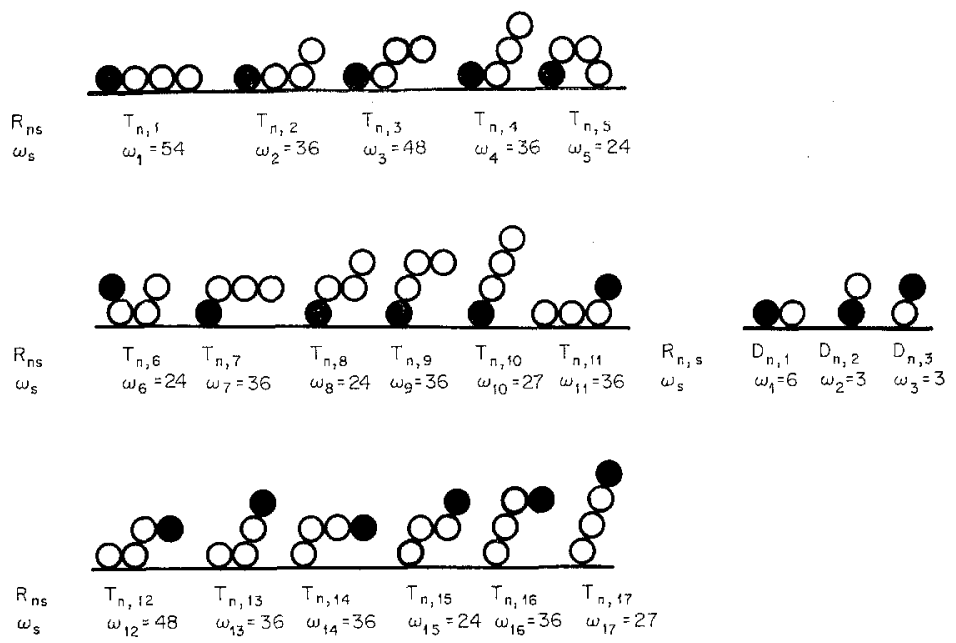

EIG. 1. Configurational species of asymmetric dimers and tetramers in a multilayer system.

of the dimer $(A-B)$ and the tetramer $(A-B-B-B){ }^{1}$

\section{EXPERIMENTAL}

The parameters that the model requires for the system of adsorbing polymers to be completely defined are:

1. Nearest neighbor energies of interaction for contacting, nonbonded segments $E_{a c}, E_{b b}, E_{c c}, E_{a b}, E_{a c}$, and $E_{b c}$, where $a$ and $b$ are segments of the polymer and $c$ represents the solvent.

2. Energies of interaction between the surface and one mole of like segments in a given $i$ th layer: $\chi A(1), \chi A(2) \cdots \chi A(i) ; \chi B(1)$, $\chi B(2) \cdots \chi \chi_{B}(i) ; \chi C(1), \chi C(2) \cdots \chi C(i)$.

3 . The bulk volume fraction of the polymer.

Once these parameters are defined, the computer is programmed to make a first guess as to the number of each polymer configuration in each layer from the first layer on the surface to, perhaps, the tenth layer, which is assumed to be far enough away from the surface to be considered in the bulk (if that assumption is invalid, it will be readily apparent from the

1 The case involving the tetramer, $A-B-B-A$, with solvent $C$ has already been investigated (3). results). Using this first guess, the chemical potential of each configuration is calculated and compared with the known chemical potential of the polymer in the bulk. If all the chemical potentials are not numerically the same, a better guess is made, and so on, until such is the case.

The computer output consists of: (1) the volume fraction of each configuration in each layer; (2) the chemical potential of the polymer; (3) the entropy per molecule of each configuration in each layer; (4) the energy per molecule of each configuration in each layer; and (5) the surface excess $(\Gamma / \nu)$ in moles per mole of surface sites, where $\Gamma$ is the total surface excess in moles per unit area and $\nu$ is the moles of surface sites per unit area.

The parameters chosen in the present study of dimers $(A-B)$ and tetramers $(A-B-B-B)$ (Fig. 1) in monomeric solvent $(C)$ are listed in Table 1.

Only representative cases and the highlights of others will be presented in this paper.

Note that a constant may be added to any set of segment-to-segment interaction energies and the results will be identical.

In this study, by varying each segment-tosegment interaction energy independently by the same amount, one not only can observe the effect that each interaction energy has, but 
TABLE I

Values of the Parameters for which Numerical Solutions Were Calculated

\begin{tabular}{clccccccccc}
\hline Case & Figure $(\mathrm{s}) \boldsymbol{a}$ & $x A^{b}(1)$ & $x B(1)$ & $x C(1)$ & $E_{A A^{b}}$ & $E_{B B}$ & $E_{C C}$ & $E_{A B}$ & $E_{A C}$ & $E_{B C}$ \\
\hline 1 & $3 A, B, C$ & -2 & 0 & 0 & 0 & 0 & 0 & 0 & 0 & 0 \\
2 & $4 A, B$ & -2 & 0 & 0 & 0 & 0 & 0.1 & 0 & 0 & 0 \\
3 & $5 A, B, C$ & -2 & 0 & 0 & 0 & 0 & 0 & 0 & 0 & 0.1 \\
4 & 6 & -2 & 0 & 0 & 0.1 & 0 & 0 & 0 & 0 & 0 \\
5 & 7 & -2 & 0 & 0 & 0 & 0.1 & 0 & 0 & 0 & 0 \\
6 & $8 A, B$ & -2 & 0 & 0 & 0 & 0 & 0 & 0 & 0.1 & 0 \\
7 & 9 & -2 & 0 & 0 & 0 & 0 & 0 & 0.1 & 0 & 0 \\
8 & 10 & -2 & 0 & 0 & 0 & 0 & 0 & 0 & 0 & -0.1 \\
9 & 11 & -2 & 0 & 0 & -0.1 & 0 & 0 & 0 & 0 & 0 \\
10 & 12 & -2 & 0 & 0 & 0 & -0.1 & 0 & 0 & 0 & 0 \\
11 & 13 & -2 & 0 & 0 & 0 & 0 & 0 & -0.1 & 0 & 0 \\
12 & 14 & -2 & 0 & 0 & 0 & 0 & -0.1 & 0 & 0 & 0 \\
13 & 15 & -2 & 0 & 0 & 0 & 0 & 0 & 0 & -0.1 & 0 \\
\hline
\end{tabular}

${ }^{a}$ Details of these calculations, equations, and computer programs are available from the authors.

${ }^{b} \mathrm{Kcal} / \mathrm{mole}$.

one also may learn that some parameters have a more profound effect on adsorption than others.

\section{RESULTS}

\section{Prefatory Remarks on the Entropy of Polymers}

It is well to make some prefatory remarks on the effects of entropy in two cases: (1) as infinite dilution is approached and (2) as the surface excess becomes such that very little solvent is left in the first layer.

The entropy of each configuration, $s$, of a polymer is $k \ln \lambda_{n s} / N_{n s}$, where $N_{n s}$ is the number of polymers of configuration $s$ in which any segment is no closer to the surface that the $n$th layer (such configuration being denoted by the expression $R_{n s}$ ), $\lambda_{n s}$ is the number of ways that configuration $R_{n s}$ can replace appropriately positioned solvent monomers and $k$ is Boltzmann's constant. For example, if there are very few monomers present, $\lambda_{n s}$ will be small. The term $k \ln \lambda_{n s}$ takes into account the straightforward fact that as the number of ways of arrangement of a species (in a unit area of the $n$th layer) increases, its entropy must become larger. However, if $\lambda_{n s}$ is small because polymers of configurations other than $s$ are present, then the entropy of configuration $s$ must be different than the case in which $\lambda_{n s}$ is small because mostly configuration $s$ is present. (In fact, in the former case, it is intuitive that the entropy must be larger than that of the latter case.) The term $-k \ln N_{n s}$, takes this into account.

The term, $\omega_{s}$, is defined as the number of ways of forming a polymer molecule of configuration $s$ (no matter what the layer) in the presence of only solvent monomer molecules. Thus, when the solution approaches infinite dilution then a polymer molecule of configuration $R_{1,1}$ and a polymer molecule of configuration $R_{1,2}$ have a ratio of $\lambda_{n s}$ values as follows :

$$
\underset{\text { concentration } \rightarrow 0}{\operatorname{limit}}\left[\frac{\lambda_{1,1}}{\lambda_{1,2}}\right]=\frac{\omega_{1}}{\omega_{2}} .
$$

It will be seen that when two configurations have identical specific thermal energies, as infinite dilution is approached, to maintain identical chemical potentials (as is required for equilibrium), their respective entropies must be identical. Consequently,

$$
\frac{\lambda_{1,1} / N_{1,1}}{\lambda_{1,2} / N_{1,2}}=1
$$


and

$$
\underset{\text { concentration } \rightarrow 0}{\operatorname{limit}}\left[\frac{N_{1,1}}{N_{1,2}}\right]=\frac{\omega_{1}}{\omega_{2}} .
$$

The consequences of this are revealed in the discussions of representative cases.

On the other hand, as the surface excess increases, $\lambda_{n s}$ will decrease more rapidly for some configurations than for others with consequences on the relative numbers of these configurations. Such an effect may clearly be observed in Fig. 2. In this case the specific thermal energy of configurations $D_{1,1}$ and $D_{1,2}$ is the same (and independent of concentration, as will be explained later), and consequently, the specific entropy of each is the same at any given bulk concentration. As the surface excess increases, the calculations reveal that it is only the first two layers that contain an excess of polymer segments and the first layer has an even greater excess than the second layer. Therefore, $\lambda_{n s}$ for a dimer of con- figuration $D_{1,1}$ will clearly decrease more rapidly than $\lambda_{n s}$ for a dimer of configuration $D_{1,2}$. Since

$$
k \ln \frac{\lambda_{1,1}}{N_{1,1}}=k \ln \frac{\lambda_{1,2}}{N_{1,2}}
$$

for all bulk concentrations and $\lambda_{1,1}$ decreases more rapidly than $\lambda_{1,2}$, then $N_{1,2}$ must be increasing more rapidly than $N_{1,1}$. Indeed, this appeals to intuition, since we are observing that when the competition for surface sites increases, this will be an advantage for configuration $D_{1,2}$ which occupies less surface and still has the same energy of attraction as configuration $D_{1,1}$.

Mackor and van der Waals (4) derived adsorption isotherms for rod-shaped polymers with one actively adsorbing end group. They also derived an expression for the ratio of the number of configuration $D_{1,2}$ to the number of configuration $D_{\mathbf{1}, \mathbf{1}}$ at the surface; however, the theory they developed can handle only rigid

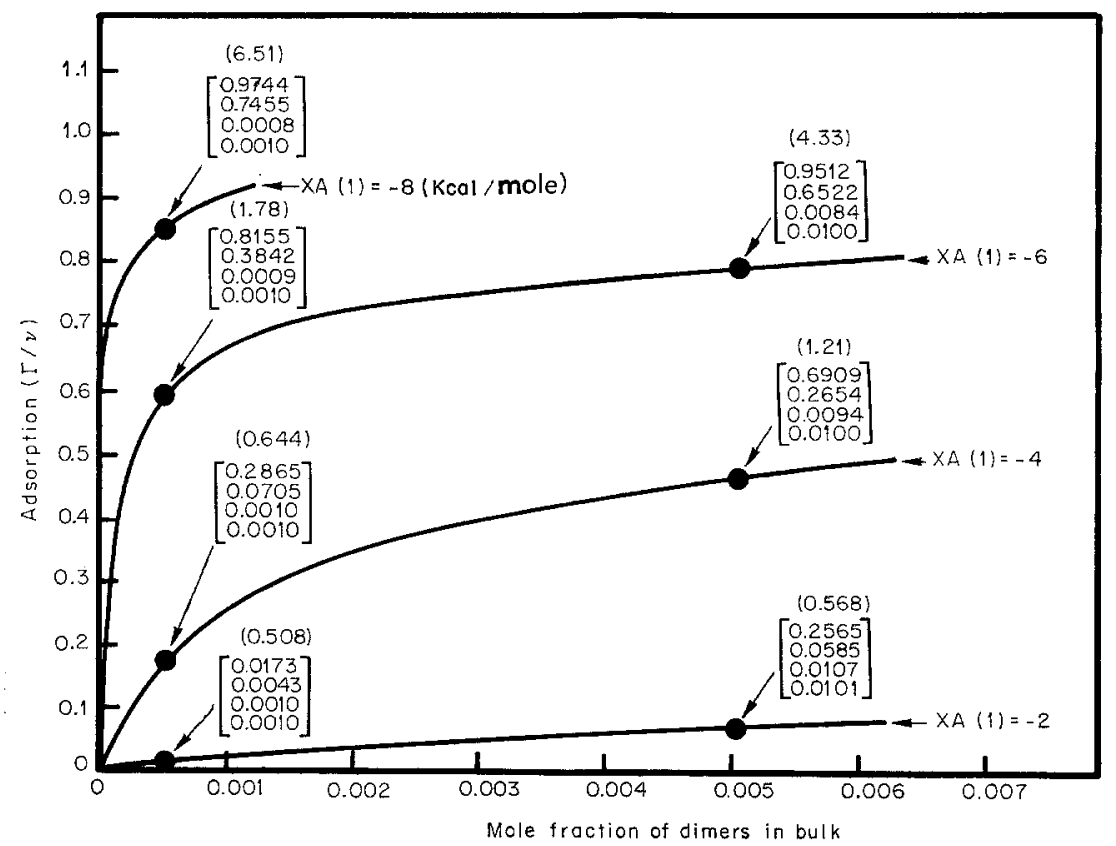

FIG. 2. The effect of $\chi A$ (1) on dimer adsorption. The numbers in parentheses are the ratios of the number with configuration $D_{1, z}$ to the number with the configuration $D_{1,1}$ at that point on the isotherm. The corresponding numbers in brackets represent the volume fraction of the polymer solute in layers $1,2,3$, and 4 (reading from top to bottom) and reveal that coverage is essentially monolayer. $E_{A A}=E_{B B}$ $=E_{C C}=E_{A B}=E_{A G}=E_{B C}$. 


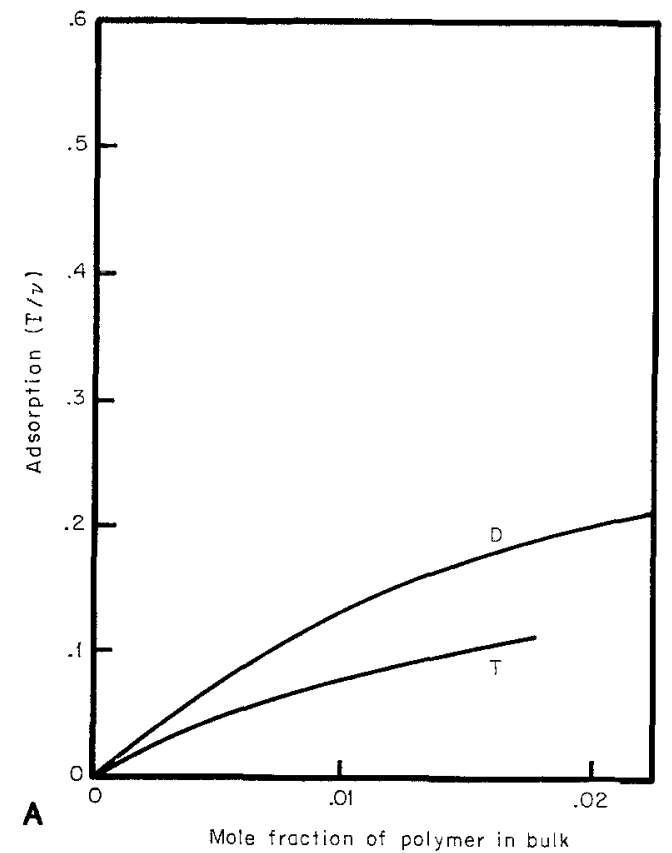

FIG. 3A. Adsorption (surface excess) curves for the dimer $(D)$ and the tetramer $(T)$ for case 1.

inflexible molecules. Their model also neglects the effect of the solution interaction energy on the configuration of the adsorbed molecules.
The results of the computations for the dimer and the tetramer are presented in graph form. For each of the cases, the following graphs are utilized: the adsorption curves, the energies per molecule of each configuration, and configuration diagrams showing the number of each configuration at the surface as a function of bulk concentration.

\section{Case 1:}

$$
\begin{aligned}
& E_{A A}=0 \quad E_{A B}=0 \\
& E_{B B}=0 \quad E_{A C}=0 \\
& E_{C C}=0 \quad E_{B C_{A}}=0 \\
& \chi A(1)=-2, \quad \chi B(1)=0, \quad \chi C(1)=0
\end{aligned}
$$

This is a special case which could be handled by the model of Ash et al. since all the segmentto-segment interaction energies are the same.

The dimer has greater surface excess than the tetramer because the surface area covered by one mole of dimer (which is one molecular layer thick) takes up less area than one mole of tetramer (see Fig. 3A).

As infinite dilution is approached, while it is true that the thermal energy portion of

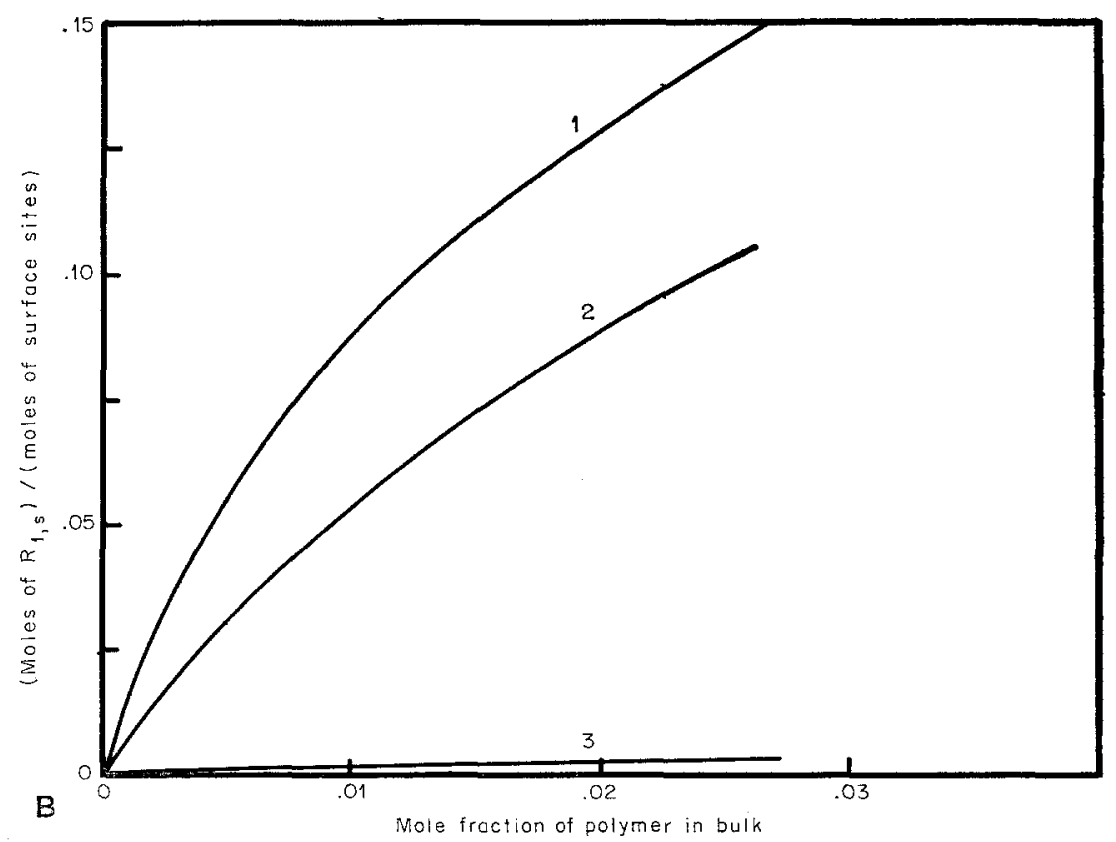

FIG. 3B. Configuration diagram for the dimer for case 1. 


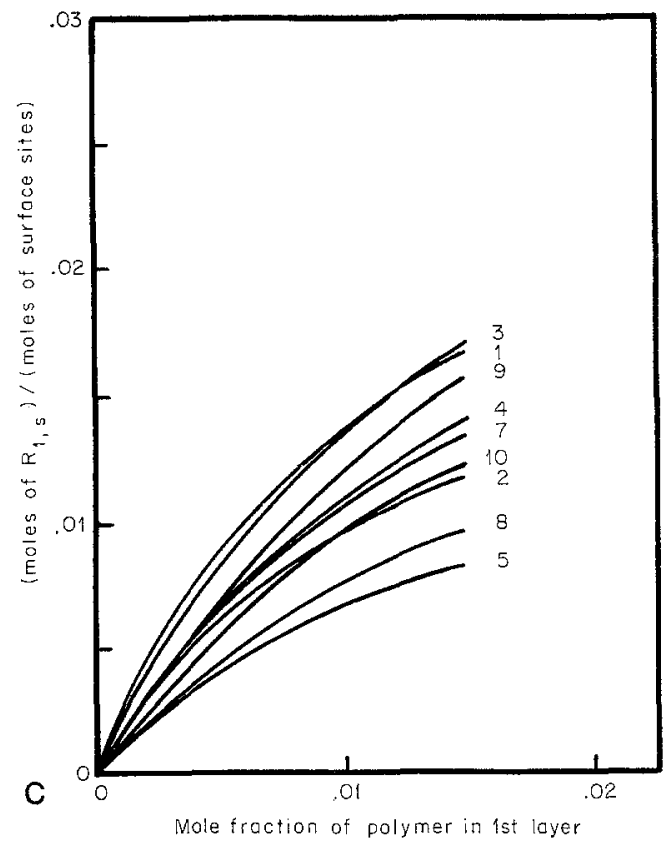

Fig. 3C. Configuration diagram for the tetramer for case 1.

the chemical potential is $-2 \mathrm{kcal}$ per mole in the first layer for the dimers and tetramers whose configurations have an $A$ segment

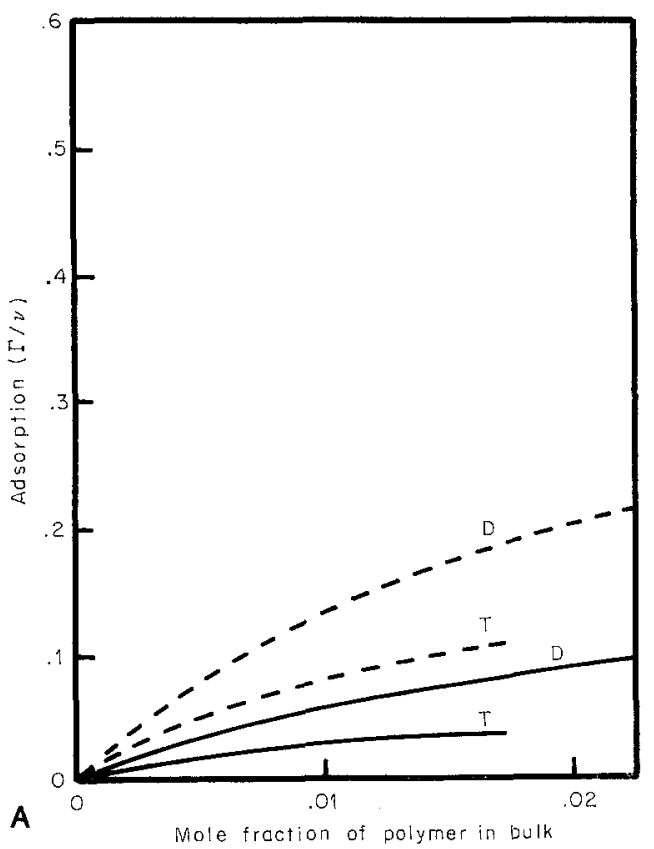

Fig. 4A. Adsorption curves for the dimer $(D)$ and the tetramer $(T)$ for case 2 (solid line). Case 1 is shown in dotted lines.

touching the surface, the chemical potentials of the two polymers are not the same-the

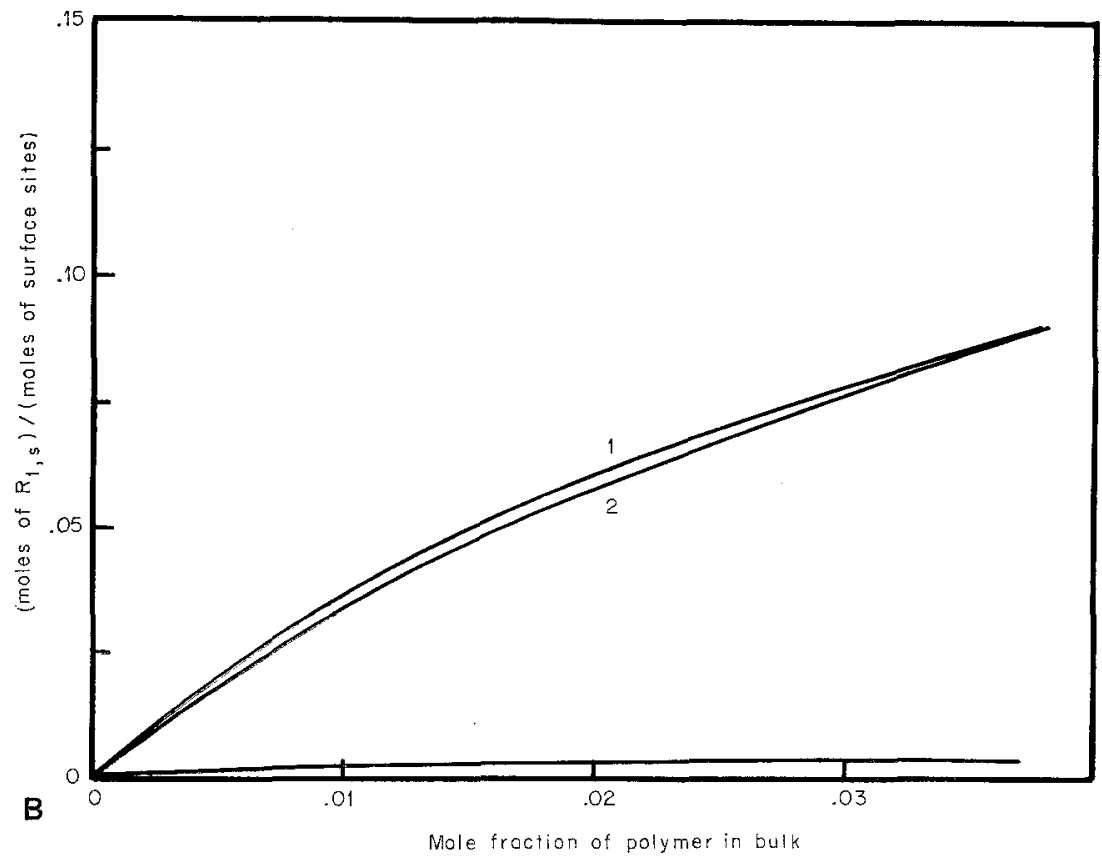

FIG. 4B. Configuration diagram for the dimer for case 2. 


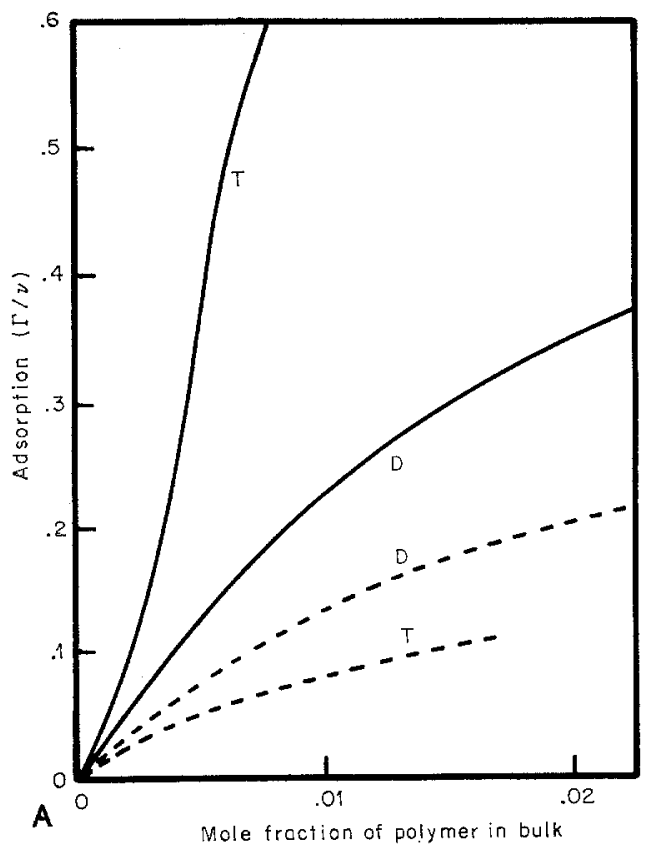

FIG. 5A. Adsorption curves for the dimer $(D)$ and the tetramer $(T)$ for case 3 (the solid lines). Case 1 is shown in dotted lines.

difference being exactly the difference between the entropy per mole of any two such configurations of dimers and tetramers.
Since the reason why the adsorption curves for the two polymers do not coincide at low concentrations may be an issue of concern, the following equation has been proven:

(initial slope of $R$-mer)

$$
=\frac{\Omega_{R}^{\prime}}{\Omega_{R}} \exp \frac{-\chi A(1)}{k N T}
$$

where $R$ is the number of segments in the $A B \cdots B$ type solute; $N$ is Avogadro's number; the segment-to-segment energies of interaction are all identical; and $\Omega_{R}{ }^{\prime} / \Omega_{R}$ represents the ratio of the number of ways of forming configurations with the $A$ segment touching the surface to the number of ways of forming all configurations. Therefore,

$$
\frac{\Omega_{2}{ }^{\prime} / \Omega_{2}}{\Omega_{4}{ }^{\prime} / \Omega_{4}}=\frac{9 / 12}{312 / 588}=1.413 \ldots
$$

Clearly, at all concentrations, if all segmentto-segment interactions are zero, then the difference in energy between a configuration with an $A$ segment touching the surface and one not touching the surface will be $2 \mathrm{kcal}$ per mole when $\chi A(1)=-2 \mathrm{kcal}$.

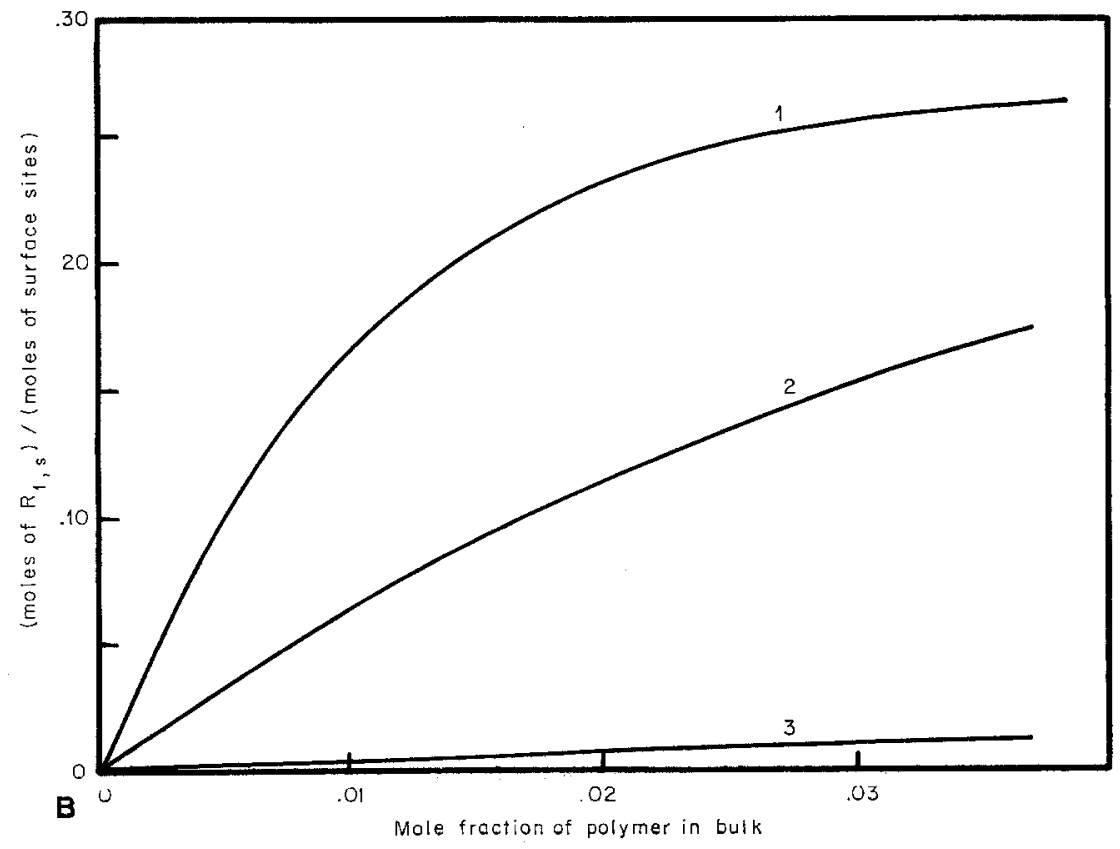

FIG. 5B. Configuration diagram for the dimer for case 3. 


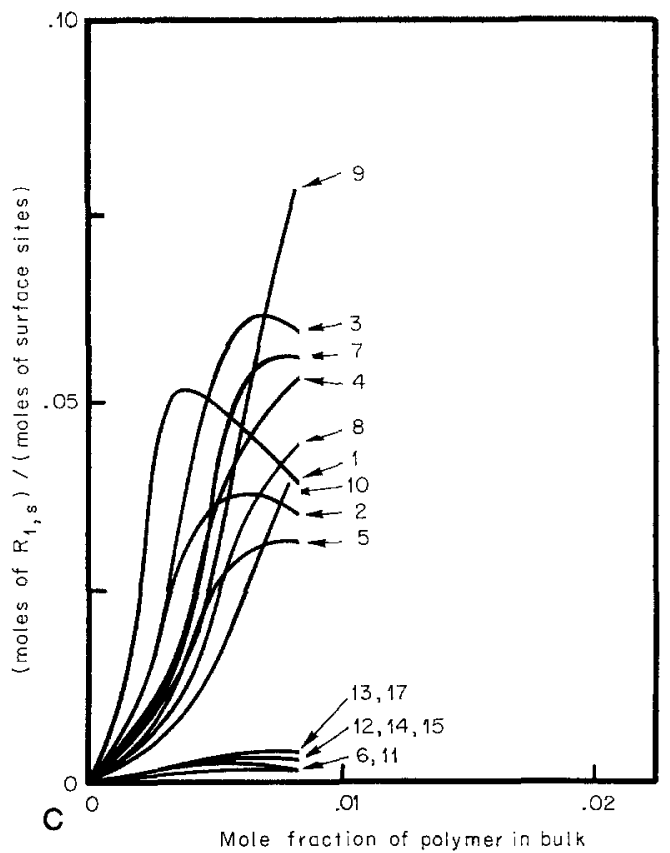

FIG. 5C. Configuration diagram for the tetramer for case 3 .

At low concentrations when all the energies of interaction are equal, the thermal portion of the chemical potential of configurations $D_{1,1}$ and $D_{1,2}$ in the first layer will be equal and the relative numbers of these two configurations will be based solely on entropy considerations (Fig. 3B). In addition, at low concentrations, the competition among the polymer molecules for surface sites becomes negligible; therefore, the entropy and consequently the difference in the chemical potential of configurations $D_{1,1}$ and $D_{1,2}$ will be determined solely by the number of ways each configuration can be formed. The result is that for species whose thermal energies are equivalent in a given layer, their relative numbers in that layer are proportional to the ratio of their $\omega_{s}$ values.

Since $\omega_{1}=6$ and $\omega_{2}=3$, then at. low concentrations, (the number with configuration $D_{1,1}$ the number with configuration $D_{1,2}$ ) $=6 / 3=2$ (see Eq. [3.3]).

As the surface excess increases, the competition for surface sites increases and the number of configuration $D_{1,2}$ rises faster than that of configuration $D_{1,1}$ because the former does not bring a $B$ segment into the first layer as does the latter, thus achieving greater utilization of surface sites for favorable surface contacts with $A$ segments. Configuration $D_{1,3}$ remains low because it does not allow its $A$ segment to contact the surface. From Fig. 2, when $\chi A(1)=-4$, it is seen that the ratio of the number with configuration $D_{1,2}$ to the number with configuration $D_{1,1}$ increases as the bulk concentration of polymer increases. (The same effect can be observed for the tetramer in case 3 , Fig. $5 \mathrm{C}$ ).

A point of interest is that at infinite dilution, tetramer configurations with the same thermal energies and $\omega_{s}$ values are equal in number at the surface (Figs. 1 and $3 \mathrm{C}$ ). These results are based on the reasoning behind $\mathrm{Eq}$. [3.3]. This series of graphs, then, forms the points of reference for comparison with the other cases.

Case 2:

$$
\begin{array}{ll}
E_{A A}=0 & E_{A B}=0 \\
E_{B B}=0 & E_{A C}=0 \\
E_{C C}=0.1 & E_{B C}=0 \\
\chi A(1)=-2, & \chi B(1)=0, \quad \chi C(1)=0
\end{array}
$$

Making $C-C$ interactions less favorable than the other segment-to-segment interactions decreases adsorption for both tetramers and dimers because solvent contact with a polymer is energetically favored over contact with other solvent molecules (Fig. 4A). The tetramer is more affected because, if a tetramer molecule must leave the surface and give up the surface interaction energy with the $A$ segment as would a dimer molecule, its presence in the bulk can eliminate more energetically unfavorable $C-C$ contacts than could the dimer molecule. Thus, some tetramer loss at the surface is not as energetically unfavorable as an equimolar amount of dimer loss from the surface.

As infinite dilution is approached, when each configuration $D_{1,3}$ or $D_{1,2}$ is brought to the 
surface and displaces two $C$ segments to the bulk, this allows three more $C-C$ contacts. When a molecule of configuration $D_{1,1}$ is brought to the surface, this allows six more $C-C$ contacts to be made in the bulk; therefore, as infinite dilution is approached, the change in energy per mole as configuration $D_{1,1}, D_{1,2}$, or $D_{1,3}$ is brought from the bulk to the first layer is $[-2+6(.1)],[-2+3(.1)]$, and $3(.1)$, or $-1.4,-1.7$, and $+0.3 \mathrm{kcal}$, respectively.

As concentration increases in the bulk, the concentration in the first two layers rises even faster, so that when an $A$ segment and a $B$ segment from their respective standard states, replace two $C$ segments at the surface (thus forming either configurations $D_{1,1}, D_{1,2}$, or $D_{1,3}$ ) which are then returned to the standard state for $C$ segments, the change in energy required for this process increases. That is, the two $C$ segments at the surface are in contact with fewer $C$ segments as the surface excess increases so that when they are returned to their standard state (in which they experience only $C-C$ contacts) the energy change grows more unfavorable. This same process with the same result may be described for the energy per molecule of configurations in the bulk, but the energy change with an increase in bulk concentration is not as great since $C-C$ contacts are lost at a slower rate in the bulk than at the surface.

Alternatively, the process of transferring a polymer from the bulk to the surface as configuration $D_{1,1}$ or $D_{1,2}$ releases less energy as the concentration increases. In this process, when the two $C$ segments are displaced from the surface to the bulk, the same argument may be used to show that this requires more energy consumption as the concentration increases. The same argument holds with respect to configuration $D_{1,3}$ except that no energy is released at any concentration since it is not actively adsorbed.

As infinite dilution is approached, the ratio of the numbers of configuration $D_{1,1}$ to those of configuration $D_{1,2}$ is not equal to the ratio of their respective $\omega_{s}$ values because their thermal energies are not equal. In fact, the thermal energy of configuration $D_{1,1}$ is greater than that of configuration $D_{1,2}$ (not the same, as in case 1); thus, the ratio of the numbers of configuration $D_{1,2}$ to the numbers of configuration $D_{1,1}$ is higher than if all the segment-to-segment contacts were the same and this continues to be true even as the surface excess increases, because the associated energy diagram shows that the energy difference between the two configurations remains about the same. Another reason for the high population of configuration $D_{1,2}$ relative to configuration $D_{1,1}$ is that the surface excess is high enough to cause $\lambda_{1,1}$ to decrease at a faster rate than $\lambda_{1,2}$. Since the thermal energy is changing at about the same rate for both configurations, the entropy of both must also change at about the same rate. For $k \ln \left(\lambda_{1,1} / N_{1,1}\right)$ to change at about the same rate as $k \ln \left(\lambda_{1,2} / N_{1,2}\right)$ when $\lambda_{1,1}$ is decreasing faster than $\lambda_{1,2}, N_{1,2}$ must increase faster than $N_{1,1}$ as surface excess increases. Accordingly, from Fig. $4 \mathrm{~B}$, it is observed that the number with configuration $D_{1,2}$ are beginning to overtake the number with configuration $D_{1,1}$.

For the tetramer there are dissimilar limiting specific thermal energies among the various configurations as infinite dilution is approached. The reason is that all configurations, when they replace monomers in the first layer, do not effect the same change in the number of $C-C$ contacts even as infinite dilution is approached. However, among those configurations which do have the same specific thermal energies ( 7 through 10 and 3 through 5), Eq. [3.3] does hold true. Among configurations that have the same $\omega_{s}$ value, those with higher specific thermal energies will be smaller in number as infinite dilution is approached.

Case 3:

$$
\begin{aligned}
& E_{A A}=0 \quad E_{A B}=0 \\
& E_{B B}=0 \quad E_{A C}=0 \\
& E_{C C}=0 \quad E_{B C}=0.1 \\
& \chi A(1)=-2, \quad \chi B(1)=0, \quad \chi C(1)=0
\end{aligned}
$$

Making $B-C$ interactions less favorable tends 


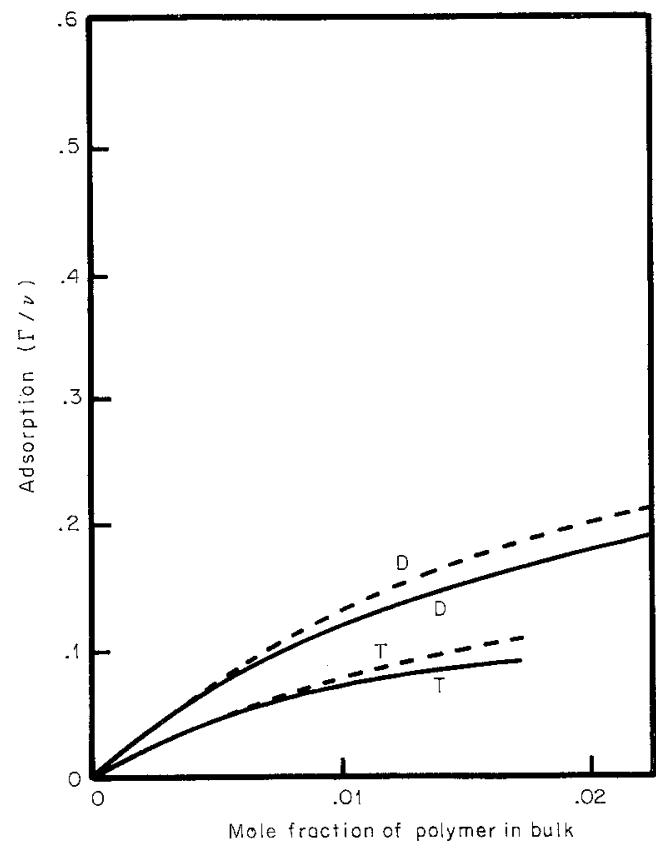

FIG. 6. Adsorption curves for the dimer $(D)$ and the tetramer $(T)$ for case 4 (the solid lines). Case 1 is shown in dotted lines.

to increase adsorption of dimers and tetramers because there are more $B-C$ contacts in the bulk than on the adsorbing surface (Fig. 5A). The tetramer is more strongly affected because in these bulk concentration ranges each tetramer in the bulk encounters more $B-C$ contacts than does a dimer in the bulk.

As infinite dilution is approached, the ratio of the numbers of configuration $D_{1,1}$ to configuration $D_{1,2}$ does not approach the ratio of their $\omega_{s}$ values, because their thermal energies, and consequently, their entropy values are not the same. At low concentrations, the ratio of configurations $D_{1,1}$ to $D_{1,2}$ (Fig. 5B) is greater than that for case 1 (Fig. 3B). The reason is that the energy per molecule is greater for configuration $D_{1,2}$ than for configuration $D_{1,1}$; whereas, for case 1 the energies per molecule are the same for these two configurations.

At higher concentrations, the ratio of the numbers of configuration $D_{1,1}$ to configuration $D_{1,2}$ become smaller even though the thermal energies of these two configurations changes at about the same rate; thus, the entropy must be playing a role. The $\lambda_{1,1}$ for configuration $D_{1,1}$ at the surface must be getting smaller at a faster rate than that for configuration $D_{1,2}$, since the number of $C$ segments disappears at a faster rate in the first layer than in the second layer as the surface excess increases.

Case 4:

$$
\begin{array}{ll}
E_{A A}=0.1 & E_{A B}=0 \\
E_{B B}=0 & E_{A C}=0 \\
E_{C C}=0 & E_{B C}=0 \\
\chi A(1)=-2, & \chi B(1)=0, \quad \chi C(1)=0
\end{array}
$$

Making $A-A$ interactions less favorable tends to decrease adsorption of both dimers and tetramers, because there are more $A-A$ contacts at the surface than in the bulk (Fig. 6). The number of $A-A$ contacts increases at a faster rate on the surface than in the bulk as the concentration in the bulk increases, because the surface excess is increasing.

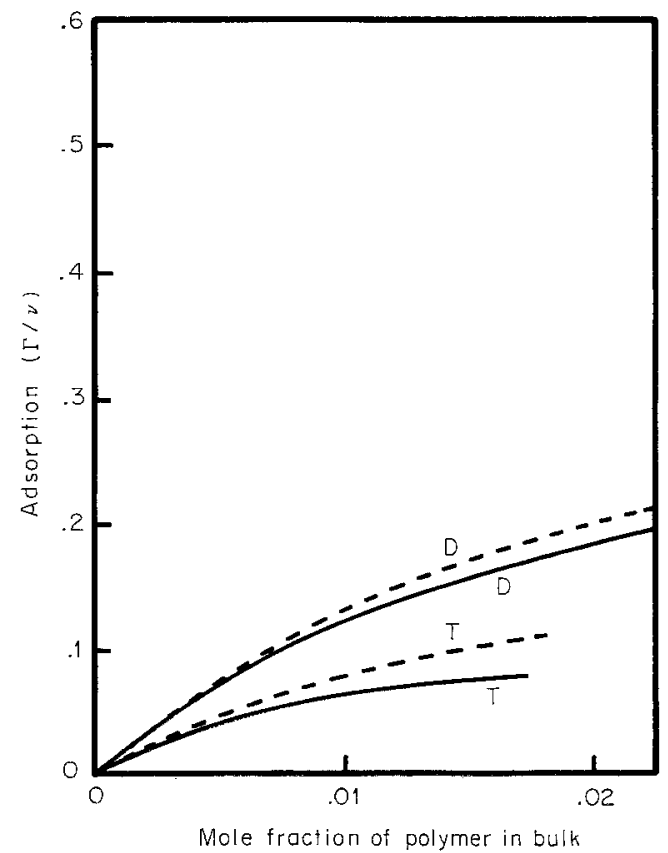

FIG. 7. Adsorption curves for the dimer $(D)$ and the tetramer $(T)$ (the solid lines) for case 5 . Case 1 is shown in dotted lines. 
Case 5:

$$
\begin{array}{ll}
E_{A A}=0 & E_{A B}=0 \\
E_{B B}=0.1 & E_{A C}=0 \\
E_{C C}=0 & E_{B C}=0 \\
\chi A(1)=-2, & \chi B(1)=0, \quad \chi C(1)=0
\end{array}
$$

Making $B-B$ interactions less favorable dedepresses adsorption of both dimers and tetramers because there are more $B-B$ contacts at the adsorbing surface than in the bulk (Fig. 7). Tetramer adsorption seems to be more affected, apparently because each tetramer brings three times as many $B$ segments with it to the surface than does the dimer.

\section{Case 6:}

$$
\begin{array}{ll}
E_{A A}=0 & E_{A B}=0 \\
E_{B B}=0 & E_{A C}=0.1 \\
E_{C C}=0 & E_{B C}=0 \\
A(1)=-2, & B(1)=0, \quad C(1)=0
\end{array}
$$

Making $A-C$ interactions less favorable increases adsorption for both dimers and tetramers because there are more $A-C$ interactions in the bulk than on the adsorbing surface (Fig. 8A).

At the surface excess increases for the dimer case, the number of $A-C$ contacts in the first layer decreases. Of the two predominant configurations $D_{1,1}$ and $D_{1,2}$ only one of them contributes even one segment to the second layer; therefore, the number of $C$ segments in the second layer does not decrease as rapidly as those in the first layer. In addition, as the surface excess increases, configuration $D_{1,3}$ does not lose as many $A-C$ contacts on arrival at the surface as do configurations $D_{1,1}$ and $D_{1,2}$, so its energy per molecule does not fall as rapidly as configurations $D_{1,1}$ and $D_{1,2}$. The $A$ segment in configuration $D_{1,1}$ has fewer contacts (five) with the first layer (which has fewer $C$ segments than the second layer) than does the $A$ segment of configuration $D_{1,2}$ (six). The former also has more contacts from the second layer with its $A$ segment (three) than does the latter (two). As a consequence, configuration $D_{1,1}$ loses fewer $A-C$ contacts on arrival at the surface. One might then expect that its energy per molecule would not fall as rapidly as would that of configuration $D_{1,2}$; however, for every configuration arriving at the surface from the bulk, two $C$ segments must take its former position in the bulk. This latter process for configuration $D_{1,1}$ should result in a greater loss of $A-C$ contacts than that for the configuration $D_{1,2}$.

As infinite dilution is approached, the ratio of the numbers of configuration $D_{1,1}$ to configuration $D_{1,2}$ is equal to the ratio of their respective $\omega_{s}$ values, again, because their thermal energies, and, consequently, their entropies are the same (Fig. 8B).

Despite the more favorable thermal energy of configuration $D_{1,1}$ as the surface excess increases, its $\lambda_{1,1}$ values become smaller at a faster rate than that of configuration $D_{1,2}$ (viz., configuration $D_{1,1}$ has more difficulty finding available space in the first layer), to the extent that the ratio of the number with configuration

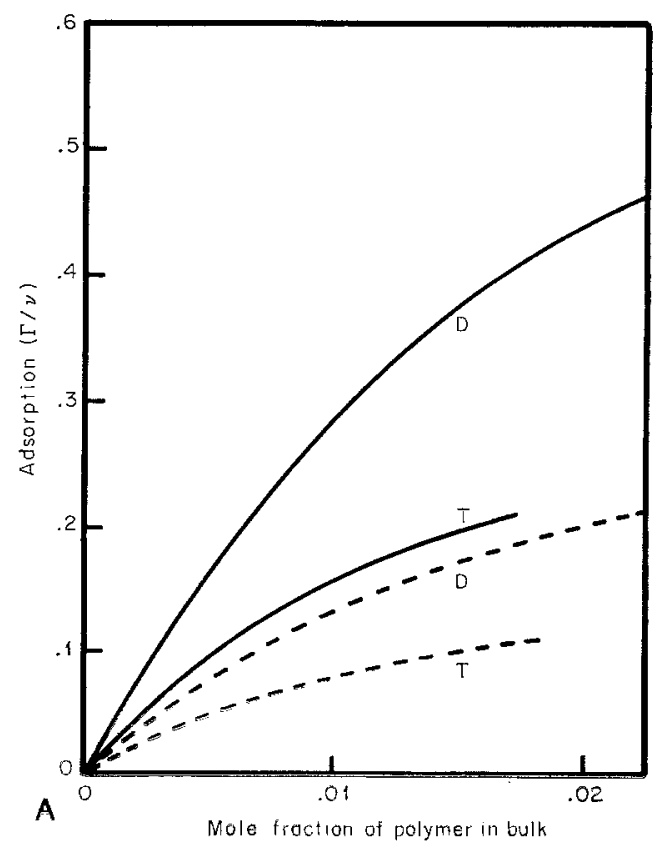

FIG. 8A. Adsorption curves for the dimer $(D)$ and the tetramer $(T)$ for case 6 (the solid lines). Case 1 is in dotted lines. 


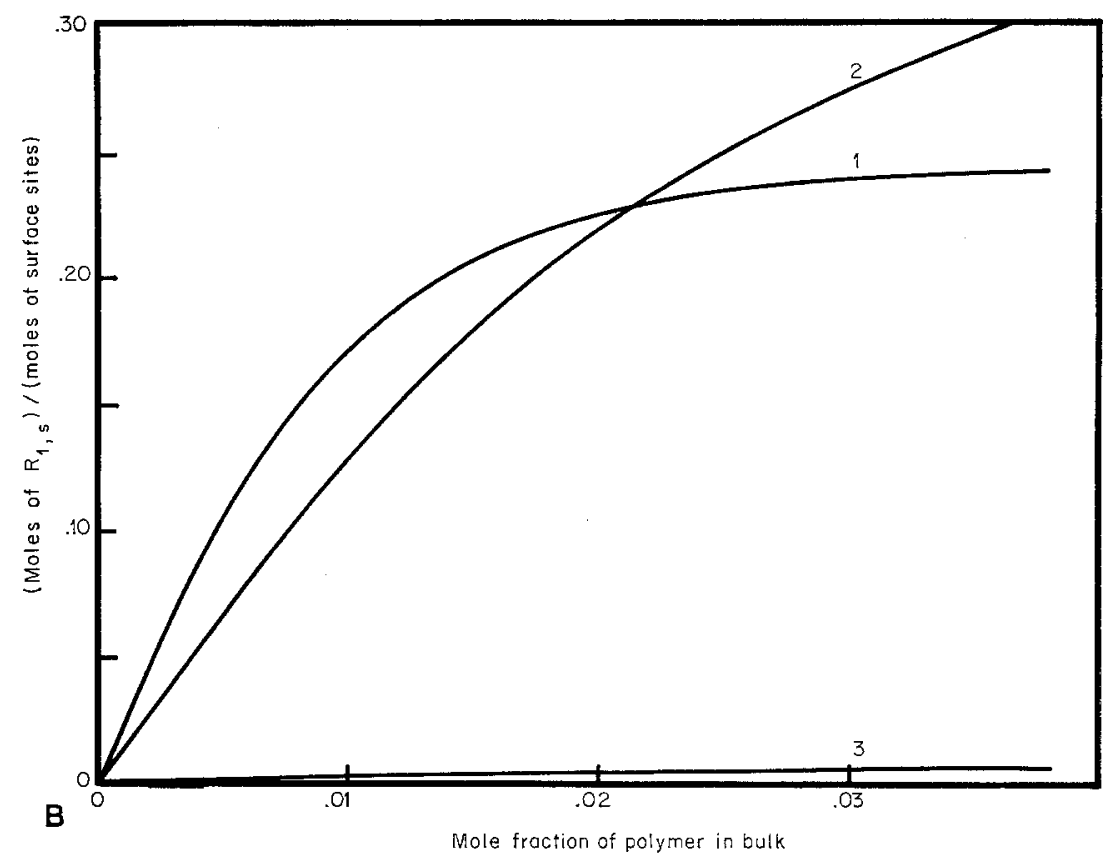

FIG. 8B. Configuration diagram for the dimer for case 6 .

$D_{1,1}$ to the number with configuration $D_{1,2}$ decreases. This effect was also observed in Fig. 2 for $\chi A(1)=-4$.

Case 7:

$$
\begin{aligned}
& E_{A A}=0 \quad E_{A B}=0.1 \\
& E_{B B}=0 \quad E_{A C}=0 \\
& E_{C C}=0 \quad E_{B C}=0 \\
& \chi A(1)=-2, \quad \chi B(1)=0, \quad \chi C(1)=0
\end{aligned}
$$

Both dimer and tetramer adsorption is depressed by making $A-B$ interactions less favorable than other segment-to-segment interactions, because there are more $A-B$ interactions at the adsorbing surface than in the bulk (Fig. 9). As the adsorption increases with increasing bulk concentration, the increasing numbers of $A-B$ contacts cause increasingly greater depression on adsorption relative to the case in which all segment-to-segment interactions are identical.

\section{Cases 8-1.3:}

Cases $8-13$ are analogous to the previous cases, except that the energy change is -0.1 $\mathrm{kcal} /$ mole, producing more favorable energies of interaction. When $B-C$ interactions are made more attractive (Fig. 10), tetramer ad-

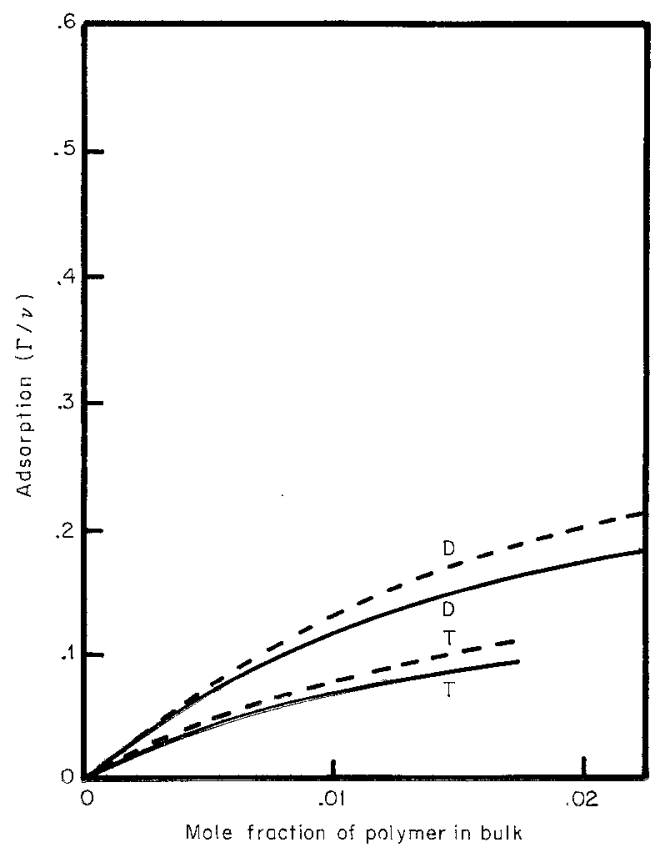

Fig. 9. Adsorption curves for the dimer $(D)$ and the tetramer $(T)$ for case 7 (the solid lines). Case 1 is in dotted lines. 


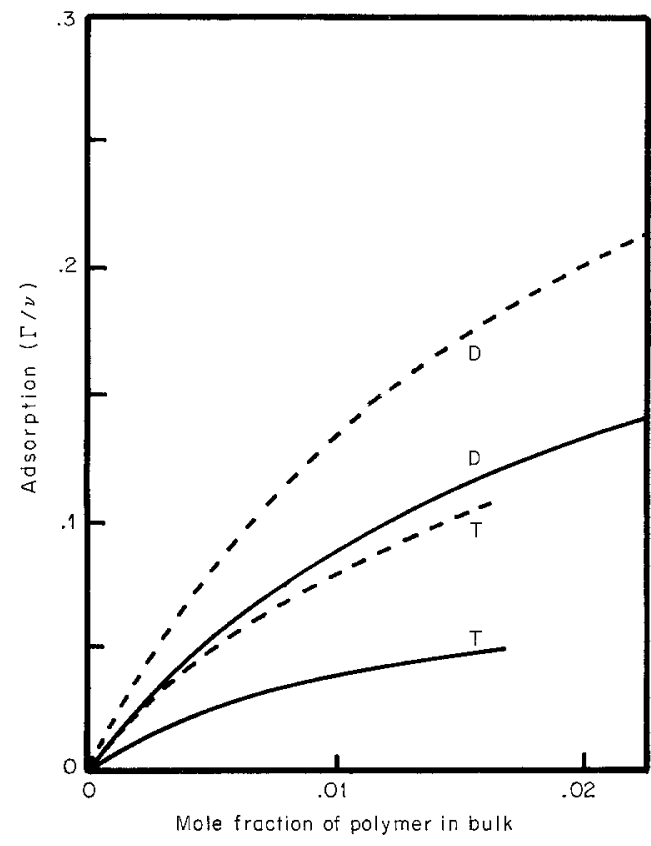

FIG. 10. Adsorption curves for the dimer $(D)$ and the tetramer $(T)$ for case 8 (the solid lines). Case 1 is in dotted lines.

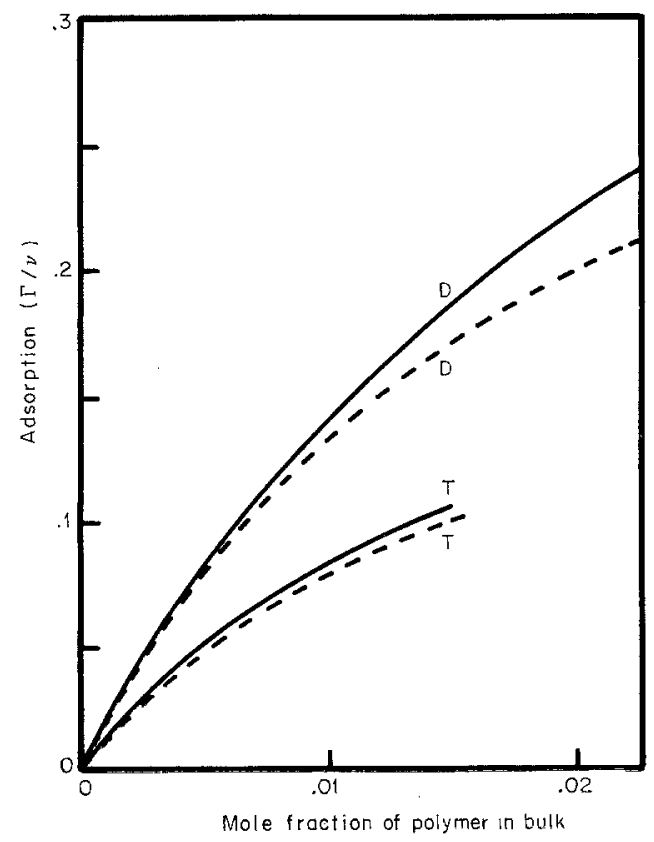

FIG. 11. Adsorption curves for the dimer $(D)$ and the tetramer $(T)$ for case 9 (the solid lines). Case 1 is in dotted lines.

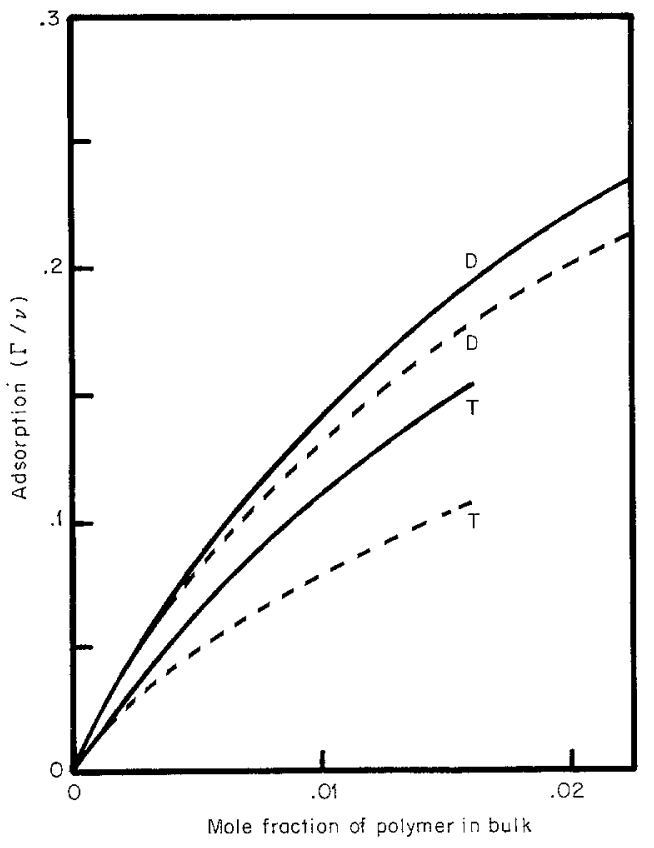

FIG. 12. Adsorption curves for the dimer $(D)$ and the tetramer $(T)$ for case 10 (the solid lines). Case 1 is in dotted lines.

sorption is depressed to a greater extent than is the dimer, since each molecule of tetramer can accommodate more $B-C$ contacts than each molecule of dimer. When $A-A$ interactions are made more attractive (Fig. 11), initial adsorption curves are identical to case 1 , since there are few $A-A$ contacts at relatively low concentrations. The dimer adsorption is increased to a greater extent than the tetramer because there are fewer $B$ segments at the surface to interfere with possible $A-A$ contacts. When $B-B$ interactions are made more attractive (Fig. 12), again it is observed that initial slopes compared to case 1 are unchanged at sufficiently low concentrations where $B-B$ contacts are few. At higher concentrations, the tetramer adsorption will increase to a greater extent, since each tetramer molecule brings more $B$ segments with it to the surface to produce energetically favorable $B-B$ contacts. When $A-B$ interactions are made more attractive (Fig. 13), the two observations that can be made are the identical initial slopes when compared to case 1 and the 


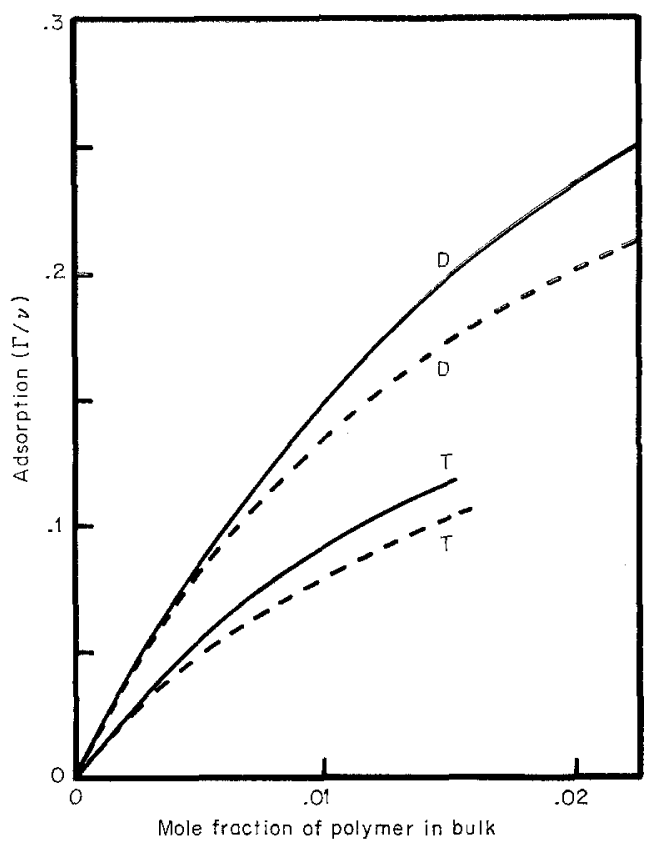

FIg. 13. Adsorption curves for the dimer $(D)$ and the tetramer $(T)$ for case 11 (the solid lines). Case 1 is in dotted lines.

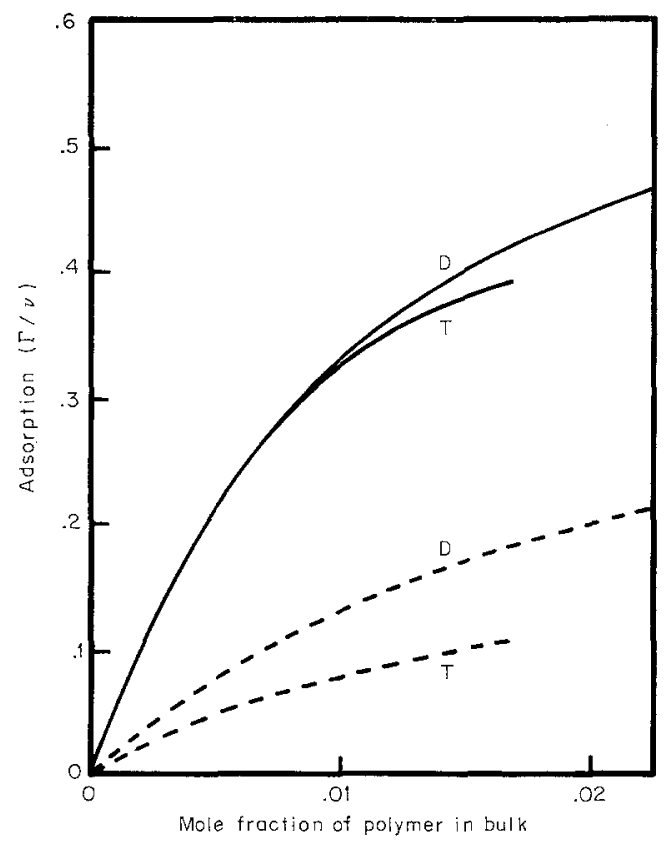

Frg. 14. Adsorption curves for the dimer $(D)$ and the tetramer $(T)$ for case 12. Case 1 is in dotted lines.

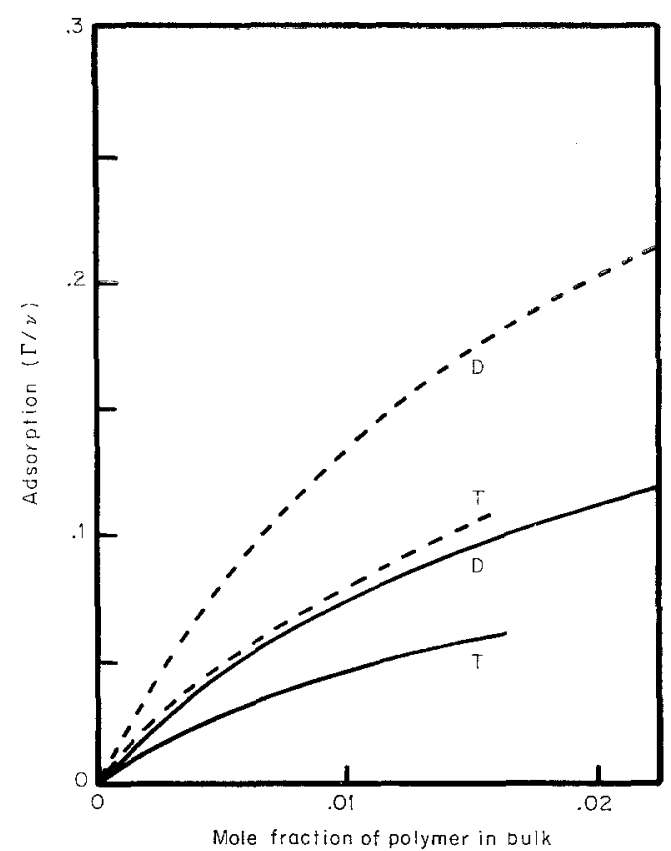

Fig. 15. Adsorption curves for the dimer $(D)$ and the tetramer $(T)$ for case 13 (the solid lines). Case 1 is in dotted lines.

increase in adsorption for both polymers. When $C-C$ interactions are made more attractive (Fig. 14, note the change in scale), adsorption is greatly increased for both polymers. Apparent identical slopes for the tetramer and dimer in Fig. 14 is a coincidence, since making $E_{C C}$ more negative will cause tetramer adsorption to be greater than dimer adsorption. The reason lies in the fact that the displacement of a four segment polymer from solvent to surface allows more $C-C$ contacts to be created than the displacement of a two segment polymer. When $A-C$ interactions are made more favorable (Fig. 15), adsorption is depressed for both polymers to approximately the same extent, since each polymer has the same number of $A$ segments.

\section{DISCUSSION}

The configuration and energy diagrams revealed that in those situations in which the amount of solvent in the first layer was sufficiently small (recognizing, of course, that what 
is "sufficiently small" cannot be determined prior to actual calculations), the differences in energies per molecule of the different configurations would no longer explain the deviations of the configuration diagrams from that for the so-called control (i.e., where all segment-to-segment interaction energies were identical); rather, it was determined that entropic considerations must be invoked. There are many ways in which such situations can be created. The most marked example was found in the configuration diagram in case 6 (Fig. 8B), in which $A-C$ interactions were made more unfavorable than others. Another situation in which the presence of solvent at the surface is sufficiently small may be produced by postulating a more negative value for $\chi A(1)$. Fig. 2 shows that as $\chi A(1)$ is made more negative, to more strongly attract $A$ segments to the surface, then the numbers of solvent molecules in the first layer is depressed more rapidly than those in the second layer. Further, it is shown that as this occurs, the ratio of the number with configuration $D_{1,2}$ to the number with configuration $D_{1,1}$ increases. One can either agree that, as $\chi_{A}(1)$ is made more negative, $\lambda_{i, 1}$ grows smaller at a faster rate than $\lambda_{1,2}$ or concede that as the presence of solvent falls off most rapidly in the first layer, then configuration $D_{1,1}$ will have the greatest difficulty in finding available surface sites for occupation.

Although the data for it are not presented here, the effect of negative values for $\chi C(1)$ may be most adequately summed up by the following observations of theoretical results:

1. Increasing negative values for $\chi C(1)$ acts to reduce polymer adsorption, as one would expect if the solvent molecules were strongly adsorbing.

2. It depresses tetramer more than dimer adsorption, apparently as a result of the greater surface area covered by significant numbers of some of the tetramer configurations ( $B$ segments are postulated to have a zero energy of interaction in this case).

These investigations have provided a means of critically studying the various interactions influencing the polymer-solvent-adsorbent surface system and further provide a base to look at more complex theoretical considerations and to evaluate experimental data on the basis of the model. Current work includes:

1. Interpretation of experimental investigations into solvent and chain length effects on the adsorption of $n$-alkyl, polar anchoring group compounds.

2. The extension of the model to multisite surfaces and to considerations of the other complex phenomena such as hydrogen bonding.

\section{ACKNOWLEDGMENTS}

This project was supported in part by NIH-NIDR Grant No. DE 01830. Grateful acknowledgment is given to Drs. S. G. Ash and D. H. Everett for supplying us with the original computer program and to Robert Pearlman for rewriting it for the IBM 360.

\section{REFERENCES}

1. Ash, S. G., Ph.D. dissertation, The University, Bristol, England, 1970.

2. Ash, S. G., Everetr, D. H., AND FindenegG, G. H., Trans. Faraday Soc. 66, 708 (1970).

3. Bladon, J. J., Moloknta, A. M., Higuchi, W. I., and Pearlman, R., to be published.

4. Mackor, E. L., AND VAN DER WAAI, J. M., $J$. Colloid Sci. 7, 535 (1952). 